\title{
Controlled Assembly of Gold Nanostructures on a Solid Substrate via Imidazole Directed Hydrogen Bonding for High Performance Surface Enhance Raman Scattering Sensing of Hypochlorous Acid
}

\author{
Jiefang Sun, Rui Liu, ${ }^{\dagger}$ Jijun Tang, ${ }^{\S}$ Zongmian Zhang, ${ }^{\dagger}$ Xiaoxia Zhou, ${ }^{\dagger}$ and Jingfu Liu*, ${ }^{*,+}$ \\ ${ }^{\dagger}$ State Key Laboratory of Environmental Chemistry and Ecotoxicology, Research Center for Eco-Environmental Sciences, Chinese \\ Academy of Sciences, P.O. Box 2871, Beijing 100085, China \\ ${ }^{\ddagger}$ Institute of Environment and Health, Jianghan University, Wuhan 430056, China \\ ${ }^{\S}$ State Key Laboratory of Toxicology and Medical Countermeasures, and Laboratory of Toxicant Analysis, Institute of Pharmacology \\ and Toxicology, Academy of Military Medical Sciences, Beijing, 100850, China
}

\section{Supporting Information}

ABSTRACT: Here, we report an efficient and facile method for constructing plasmonic gold nanostructures with controlled morphology on a Si wafer and its use as a surface enhanced Raman scattering (SERS) reporting system for specific detection of $\mathrm{HClO}$. To achieve this substrate, the core gold nanoparticles (AuNPs, $\sim 100 \mathrm{~nm}$ ) with a monolayer of 4mercaptoimidazole (MI) ligands were covalently linked to a thiol-derived Si wafer (MI-AuNPs@SH-Si). Taking advantage of the intermolecular $\mathrm{NH} \cdots \mathrm{N}$ hydrogen bond ( $\mathrm{HB}$ ) provided by the neighboring imidazole moiety, multiple satellite AuNPs $(\sim 12 \mathrm{~nm})$ decorated with both MI and a Raman reporter are assembled around the core MI-AuNPs at $\mathrm{pH}$ 5.0. The uniform morphology of the AuNP-based nanostructures on the Si wafer offer a high density of hot spots with good SERS performance for detecting $\mathrm{HClO}$. The fast oxidation of the imidazole moieties by $\mathrm{HClO}$ causes $\mathrm{HB}$ destruction and therefore separation of the satellite AuNPs from the core AuNPs, which gives rise to SERS signal damping of the chip that is employed for $\mathrm{HClO}$ analysis. This simple and cost-effective method is highly selective for $\mathrm{HClO}$ over common interferences and several reactive oxygen/nitrogen species, and enabled rapid analysis at concentrations as low as $1.2 \mu \mathrm{mol} \mathrm{L} \mathrm{L}^{-1}$. The present approach is applied to analyze water and human serum samples with satisfactory results.

KEYWORDS: surface-enhanced Raman scattering, hypochlorous acid, gold nanoparticles, imidazole, hydrogen bonding

\section{INTRODUCTION}

Hypochlorous acid $\left(\mathrm{HClO}, \mathrm{p} K_{\mathrm{a}}=7.46\right)$ is approximately half dissociated into the hypochlorite anion under physiological conditions and is one of the most powerful natural reactive oxygen species (ROS). ${ }^{1}$ Endogenous $\mathrm{HClO}$, which is produced mainly in leukocytes, including neutrophils, macrophages and monocytes, by myeloperoxidase (MPO)-catalyzed peroxidation of chloride ions, ${ }^{2}$ likely injures invasive microorganisms and is involved in the chemical modification of biomolecules. ${ }^{3,4}$ However, it can also exert a detrimental effect on host tissues ${ }^{5}$ and is implicated in many diseases (e.g., atherosclerosis, kidney disease, and some cancers) by triggering oxidative stress. ${ }^{6-8}$ Therefore, feasible methods for accurately and sensitively monitoring $\mathrm{HClO}$ levels in biological samples are of great interest for elucidating the role of $\mathrm{HClO}$ in various biological processes.

Common analytic methods such as electrochemistry ${ }^{9}$ and ion chromatography ${ }^{10,11}$ offer excellent analytical performance for the determination of $\mathrm{HClO}$. However, these techniques require complex sample preparation and operation, which restrict their use in field rapid analysis. Accordingly, most research has focused on developing chromogenic ${ }^{12,13}$ and fluorogenic ${ }^{14,15}$ chemodosimeters for the detection of $\mathrm{HClO} / \mathrm{ClO}^{-}$based on its strong oxidation properties. Recently, a rhodamine-modified upconversion nanoparticles have developed for $\mathrm{HClO}$ detection in living system. ${ }^{16}$ These systems offer intuitive signal feedback and facile quantification in authentic aqueous solution or biological samples. Nevertheless, the development of more effective and reliable systems that are simple and economical to prepare and provide rapid and sensitive signal feedback with high specificity in real-world samples remains a worthwhile pursuit.

Driven by recent advances in nanofabrication, many previous limitations of surface-enhanced Raman scattering (SERS) have been overcome and this technique is emerging as one of the most promising analytical techniques. As an appealing and nondestructive technique, SERS offers enhanced sensitivity, simplified instrumentation, cost savings, and high throughput

Received: May 23, 2015

Accepted: July 13, 2015

Published: July 13, 2015 
capacity with no (or very little) need for sample handing. With these advantages, SERS is capable of addressing the everincreasing need for new types of analytical tools. ${ }^{17}$ To date, SERS has yielded the unequivocal identification of a wide of range analytes, from biomolecules ${ }^{18-21}$ and food contaminants $^{22}$ to pollutants ${ }^{23}$ and explosives. ${ }^{24}$ Despite these achievements, there have been limited research on the detection of inorganic species and their ions by SERS, ${ }^{25}$ and the relevant works mainly focus on the detection of protons $(\mathrm{pH})^{26}$ and some toxicologically defined metal ions, such as $\mathrm{As}^{3+}, \mathrm{Hg}^{2+}, \mathrm{Pb}^{2+}$, and $\mathrm{Cd}^{2+} .{ }^{27-30}$ However, the detection of ions using SERS remains largely unexplored. ${ }^{31-33}$ This can be attributed to (i) SERS substrates are made from noble metal nanoparticles of low chemical reactivity that often lack the ability to bind anions, and (ii) many anionic systems have small Raman scattering cross-section or even no vibrational modes. ${ }^{34}$ The decoration of noble metal surfaces with functional molecules that could selectively respond to inorganic species and thus cause detectable changes in the SERS signal might meet this challenge.

In this study, we fabricated special SERS-active substrates for the detection of $\mathrm{HClO}$ in different matrices. As illustrated in Scheme 1, the SERS chip comprises a monolayer of 4-

Scheme 1. Illustration of the General Design of the SERS Chip and the Sensing Procedure for HClO: (1) Modification of the Satellite AuNPs with MI and MBT ligands; (2) Assembly of the Core AuNPs on a SH-Si Wafer and Modification with MI; (3) Conjugation of the Satellite MI/ MBT-AuNPs on MI-AuNPs@SH-Si via HB Interactions; (4) Detachment of the Satellite MI-AuNPs from MI-AuNPs@ SH-Si Induced by HCIO Oxidation; (5) SERS Signal Collection after Washing the SERS Chip Post Reaction

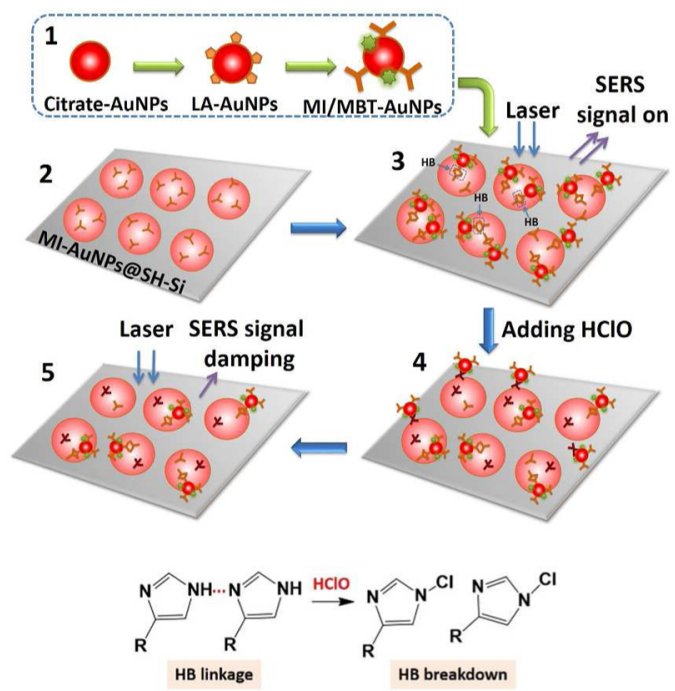

mercaptoimidazole (MI)-modified core AuNPs ( 100 nm) that is covalently arranged on a thiol-derived $\mathrm{Si}$ wafer $(\mathrm{SH}-\mathrm{Si})$. These NPs are decorated with satellite MI-modified AuNPs $(\sim 12 \mathrm{~nm})$ via an intermolecular hydrogen bond (HB) linkage. This gold nanostructure induces abundant in-built plasmonic "hot spots" that provide a significant SERS enhancement from the embedded Raman reporter (4-methylbenzenethiol, MBT). The introduction of $\mathrm{HClO}$ disrupts the $\mathrm{HB}$ linkage by immediately reacting with imidazole, ${ }^{35}$ leading to the detachment of the satellite AuNPs from the core AuNPs and consequent SERS signal damping owing to the absent of "hot spots". Therefore, the amount of $\mathrm{HClO}$ can be quantified by direct comparison of the signal change. Finally, detection of $\mathrm{HClO}$ in environmental water and human serum using this SERS chip was performed to demonstrate its application in real samples.

\section{EXPERIMENTAL PROCEDURES}

2.1. Chemicals. MI, MBT, 3-mercaptopropyl-trimethoxysilane (MPTMS), lipoic acid (LA), sodium hypochlorite solution ( $\mathrm{NaClO}$, $\sim 10 \%$ ), N, $\mathrm{N}^{\prime}$-diisopropylethylamine (DPEA), S-nitroso-N-acetyl-DLpenicillamine, 2,2'-azobis (2-methylpropionamidine) dihydrochloride (AAPH), and tert-butyl-hydroperoxide (TBHP) were purchased from Sigma-Aldrich (St. Louis, MO). 3-Morpholinosydnonimine (SIN-1), Angeli's salt $\left(\mathrm{N}_{2} \mathrm{Na}_{3} \mathrm{O}_{3}{ }^{+}\right)$were products of Cayman (Michigan, USA). $\mathrm{HAuCl}_{4}, \mathrm{KO}_{2}, \mathrm{FeSO}_{4}, \mathrm{NaMoO}_{4}$ and $\mathrm{H}_{2} \mathrm{O}_{2}$ solution (30\%) were provided from Sinopharm Chemical Reagent Co. (Beijing, China). Various ROS/RNS solutions were prepared daily, and their concentrations were determined using their absorbance immediately before use. Highly polished single-crystal silicon wafers were obtained from Wacker Chemtronics (Germany). Ultrapure water (18.3 $\mathrm{M} \Omega$ $\mathrm{cm}$ ) produced with a Milli-Q gradient system (Millipore, Billerica, MA) was used in all experiments.

2.2. Preparation of the MI/MBT-Functionalized AuNPs. First, uniform AuNPs of $\sim 12 \mathrm{~nm}$ were prepared through the citratemediated reduction of $\mathrm{HAuCl}_{4}$ in aqueous solution. ${ }^{36}$ Then, an ethanolic solution of LA $\left(1.0 \mathrm{~mL}, 0.1 \mathrm{~mol} \mathrm{~L}^{-1}\right)$ was added into the prepared citrate-AuNPs $\left(200 \mathrm{~mL}, 10 \mathrm{nmol} \mathrm{L}{ }^{-1}, \mathrm{pH} 9.0\right)$ and stirred overnight $(12 \mathrm{~h})$ to prepare the LA-capped AuNPs (LA-AuNPs). ${ }^{37}$ To purify the LA-AuNPs, we centrifuged the solution for $20 \mathrm{~min}$ at 15700 $g$ and the supernatant was decanted. The NPs were then resuspended in water. After adjusting the $\mathrm{pH}$ of the LA-AuNP solution $(10 \mathrm{nmol}$ $\left.\mathrm{L}^{-1}, 200 \mathrm{~mL}\right)$ with $\mathrm{NaOH}\left(0.1 \mathrm{~mol} \mathrm{~L}^{-1}\right)$ to $9.0,1.0 \mathrm{~mL}$ of the ethanol solution containing MBT $\left(0.1 \mathrm{~mol} \mathrm{~L}^{-1}\right)$ and $\mathrm{MI}\left(0.05 \mathrm{~mol} \mathrm{~L}^{-1}\right)$ was added. By controlling the incubation time at a temperature below 50 ${ }^{\circ} \mathrm{C}$, different $\mathrm{MI} / \mathrm{MBT}$ coverage ratios on AuNPs can be achieved. After reaction, the MI/MBT-AuNPs were purified by centrifugation (10000 $\mathrm{g}$ for $20 \mathrm{~min}$ ), washed with ethanol and water sequentially, and finally redispersed in water $\left(10 \mathrm{nmol} \mathrm{L} \mathrm{L}^{-1}\right)$ for later use. The concentration of AuNPs can be calculated according to Beer's law. ${ }^{38}$ The UV-vis absorbance of these AuNPs with different modifications were characterized using a Shimadzu 3600 UV-vis-NIR spectrometer. The morphologies of the AuNPs were observed using transmission electron microscopy (TEM, JEOL model 1200EX), and their zeta potentials were measured using a Malvern Nano ZS instrument with a $633 \mathrm{~nm}$ laser diode. To verify the successful binding of MI/MBT on the AuNPs, we conducted Raman experiments using a Renishaw InVia Raman spectrometer with a $785 \mathrm{~nm}$ laser for excitation (detailed experimental conditions are listed in Supporting Information).

2.3. Preparation of the MI-Modified AuNPs@SH-Si Substrate. First, a $3.0 \times 3.0 \mathrm{~mm}^{2} \mathrm{Si}$ wafer was chemically modified with MPTMS to obtain dangling thiols for AuNP binding (SH-Si, see the Supporting Information). Different sized AuNPs $(\sim 60 \mathrm{~nm}, \sim 75 \mathrm{~nm}$, $\sim 90 \mathrm{~nm}$ and $\sim 100 \mathrm{~nm}$ ) were prepared according to a kinetically controlled seeded growth method. ${ }^{39}$ These AuNPs were used for fabricating MI-AuNPs@SH-Si as the following steps: $0.2 \mathrm{~mL}$ of the citrate-AuNPs $\left(40 \mathrm{nmol} \mathrm{L}^{-1}\right)$ was incubated with $\mathrm{SH}-\mathrm{Si}$ in a 96 -hole plate at room temperature for $12 \mathrm{~h}$ to obtain a dense layer of AuNPs on SH-Si (AuNPs@SH-Si). After thorough rinsing with water, the AuNPs@SH-Si surface was modified with MI ligands by immersion in a $10 \mathrm{mmol} \mathrm{L}{ }^{-1} \mathrm{MI}$ aqueous solution for $5 \mathrm{~h}$; this promoted partial coverage of $\mathrm{MI}$ on the exposed surface of the AuNP array (MIAuNPs@SH-Si). Finally, MI-AuNPs@SH-Si was thoroughly rinsed with water to remove any physisorbed molecules, dried with a gentle flow of $\mathrm{N}_{2}$, and stored at $4{ }^{\circ} \mathrm{C}$ in a refrigerator for later use. The morphology of MI-AuNPs@SH-Si was observed on a field-emission scanning electron microscopy images (FESEM, Hitachi SU-8020). 
The modification of MI on AuNPs@SH-Si was also confirmed by Raman spectroscopy.

2.4. Assembly of the Satellite AuNPs around MI-AuNPs@SHSi via HB Linkages. The SERS chip was fabricated by incubating each MI-AuNPs@SH-Si substrate with $0.2 \mathrm{~mL}$ of the MI/MBTAuNPs $\left(10 \mathrm{nmol} \mathrm{L}^{-1}\right)$ dispersed in $0.01 \mathrm{~mol} \mathrm{~L}^{-1}$ sodium phosphate buffer ( $\mathrm{pH} 5.0)$ in a 96-hole plate. At different times, the resultant chip was taken out and rinsed with the water for SEM and SERS analyses. To demonstrate the stability of the chip in biological media, SERS spectra of the chip incubated in human serum diluted 50 times with sodium phosphate buffer $\left(1.0 \mathrm{mmol} \mathrm{L}^{-1}, \mathrm{pH} 6.0\right)$ were recorded at different time intervals.

2.5. Evaluation of the Analytical Performance. A standard curve for $\mathrm{HClO}$ detection was prepared as follows: various concentrations of $\mathrm{NaClO}(100 \mu \mathrm{L})$ in sodium phosphate buffer $(1.0$ mmol L $\mathrm{L}^{-1}, \mathrm{pH}$ 6.0) were added to each hole of a 96-hole plate containing the SERS chip. After reaction for $40 \mathrm{~min}$, the chip was taken out and dried under a $\mathrm{N}_{2}$ flow. Subsequently, SERS spectrum of the chip was collected. The chip was also treated with a wide variety of interfering species to evaluate its selectivity. Solutions for testing anions were prepared by dissolving their corresponding sodium salts in water, whereas solutions for testing cation interference were prepared using their corresponding nitrates. Various ROS/RNS species were freshly prepared as stock solutions $\left(5.0 \mathrm{mmol} \mathrm{L}^{-1}\right.$, see the Supporting Information). Each of these solutions $\left(100 \mu \mathrm{L}, 1.0 \mathrm{mmol} \mathrm{L}^{-1}\right)$ was dispersed in sodium phosphate buffer $\left(1.0 \mathrm{mmol} \mathrm{L}^{-1}, \mathrm{pH} 6.0\right)$ and added to a 96-hole plate containing the SERS chip, and SERS spectra were acquired after $30 \mathrm{~min}$ of incubation.

2.6. Determination of $\mathrm{HClO}$ in Real Samples. Freshly collected tap water samples were used with no pretreatment. The tap water samples $(100 \mu \mathrm{L})$ dispersed in sodium phosphate buffer $(1.0 \mathrm{mmol}$ $\mathrm{L}^{-1}, \mathrm{pH}$ 6.0) were tested using the same procedure as described above. The total content of $\mathrm{HClO}$ in tap water was then calculated based on the standard curve obtained in buffer. To detect $\mathrm{HClO}$ in human serum, we added $1.0 \mathrm{~mL}$ of acetonitrile into $5.0 \mathrm{~mL}$ of human serum and shaken vigorously to precipitate proteins. The insoluble material was removed by centrifugation (3000 $\mathrm{g}$ for $20 \mathrm{~min}$ ) and the supernatant was then diluted 50 times with sodium phosphate buffer (1.0 $\mathrm{mmol} \mathrm{L}^{-1}, \mathrm{pH} \mathrm{6.0).} \mathrm{The} \mathrm{subsequent} \mathrm{testing} \mathrm{procedure} \mathrm{was} \mathrm{the}$ same as described above. To validate this method, the recoveries obtained in real samples using this method were evaluated by spiking with different amounts of $\mathrm{NaClO}$ (Table 1).

Table 1. Determination of HClO in Tap Water and Human Serum Using the Proposed SERS Chip

\begin{tabular}{cccc} 
sample & spiked $\left(\mu \mathrm{mol} \mathrm{L}^{-1}\right)$ & detected $\left(\mu \mathrm{mol} \mathrm{L}^{-1}\right)$ & recovery (\%) \\
tap water 1 & 0 & 1.7 & \\
& 10.0 & 12.1 & 104 \\
tap water 2 & 30.0 & 32.4 & 102 \\
& 0 & 2.3 & \\
human serum & 10.0 & 12.6 & 103 \\
& 30.0 & 32.6 & 101 \\
\multirow{4}{*}{ abelow the detection limit. } & 0 & $\mathrm{nd}^{a}$ & \\
\hline
\end{tabular}

\section{RESULTS AND DISCUSSION}

3.1. Preparation and Characterization of AuNPs Modified with MI/MBT. In this work, spherical AuNPs of different sizes were synthesized and modified with functional ligands for the construction of a SERS chip. First, the satellite AuNPs $(\sim 12 \mathrm{~nm})$ were functionalized with a mixed monolayer of MI and MBT via ligand exchange. MBT was used as the SERS reporter and MI functioned as both the internanoparticle
HB linker and the specific recognition module for reaction with HClO. Although we expected MI/MBT ligands to be easily anchored on AuNPs via Au-thiol binding, the citrate-AuNPs were found to aggregate immediately in water $(10 \mathrm{~mL}, 10 \mathrm{nmol}$ $\left.\mathrm{L}^{-1}, \mathrm{pH} 9.0\right)$ when an ethanol solution of MI/MBT with molar ratio of 1:2 $\left(0.2 \mathrm{~mL}, 0.1 \mathrm{mmol} \mathrm{L}^{-1}\right.$ for $\left.\mathrm{MI}\right)$ was introduced. Therefore, a two-step ligand exchange process was adopted (as shown in Step 1 in Scheme 1). In this process, the capping agents from the initial synthesis, i.e., citrate, are substantially displaced by LA via its disulfide bond, and then LA is partially replaced by MI/MBT ligands with the help of heating, owing to the stronger Au-thiol binding of the latter. ${ }^{36}$ After modification, we characterized these AuNPs with various tools. Figure 1A, B shows the TEM images of the AuNPs, in which the AuNPs are uniformly dispersed with a narrow size distribution. The diameter and morphology of the MI/MBT-AuNPs showed little change compared to those of the precursor citrate-AuNPs. After conjugation with the MI/MBT ligands, a red shift $(\sim 7$ $\mathrm{nm}$ ) of the AuNP surface plasmon absorption (Figure 1D) was observed, suggesting that the thiol ligand conjugation locally changed the dielectric constant near the AuNP surface. Moreover, the absence of broadening or tailing of the plasmon absorption peak indicated that no aggregation occurred during the conjugation. The final colloidal solution was stable for at least 3 months. Furthermore, the SERS spectrum of the resultant MBT/MI-AuNPs is compared with those of the corresponding AuNPs modified with only MI or MBT ligands (Figure 1E). For the MI/MBT-AuNPs, the Raman vibrations corresponding to MBT are prominent, which are dominated by characteristic bands such as $\mathrm{C}-\mathrm{S}$ vibration $\left(491 \mathrm{~cm}^{-1}\right)$, phenyl ring breathing mode II $\left(691 \mathrm{~cm}^{-1}\right)$, and phenyl ring breathing vibration $\left(1078 \mathrm{~cm}^{-1}\right)$. However, the characteristic SERS peaks of MI, including $\mathrm{C}-\mathrm{S}$ vibration $\left(491 \mathrm{~cm}^{-1}\right), \mathrm{CH}_{2}$ bending (1227 $\left.\mathrm{cm}^{-1}\right), \mathrm{C}-\mathrm{N}$ vibration $\left(1318 \mathrm{~cm}^{-1}\right)$, and $\mathrm{C}-\mathrm{N}$ stretching $\left(1424 \mathrm{~cm}^{-1}\right)$, are not very prominent, which is attributed to the inherent small Raman cross section of this ligand. This result indicates that both MBT and MI ligands are successfully assembled on the AuNP surface.

To achieve a monodisperse MI/MBT-AuNP colloid, efficient control over the assembly of MI/MBT ligands on the AuNPs is required. Considering that the MI ligand has a constituent imidazole unit with a net positive charge in aqueous medium in mildly basic and acidic solutions, the binding of MI to AuNPs leads to continual reduction of surface negative charges, which, in turn, promotes AuNP aggregation. On the other hand, the low polarity of the MBT ligand could compromise the stability of the AuNPs when assembled on the Au surface. Thus, excessive replacement of the negatively charged LA ligand, which provides necessary electrostatic repulsion to AuNPs, should be avoided. A kinetic study was carried out to record the time-dependent change in the dispersion of the AuNPs after exposure to the $\mathrm{MI} / \mathrm{MBT}$ solution by monitoring the ratio of the absorbance at 630 and $530 \mathrm{~nm}\left(A_{630} / A_{530}\right)$. As shown in Figure 2A, the AuNPs were stable for the first $40 \mathrm{~min}$ of the experiment, then the $A_{630} / A_{530}$ value began to increase, suggesting the formation of aggregates owing to a decrease of the inter-NP electrostatic repulsion to a critical level that is not sufficient to maintain monodisperse AuNPs. This assertion was also confirmed by the increase of the zeta potential from -24.2 to $-3.8 \mathrm{mV}$ during the ligand exchange process. Thus, we chose an incubation time of $40 \mathrm{~min}$ as the optimized condition.

Next, we investigated the stability of the resultant MI/MBTAuNPs under different $\mathrm{pH}$ conditions. As mentioned 

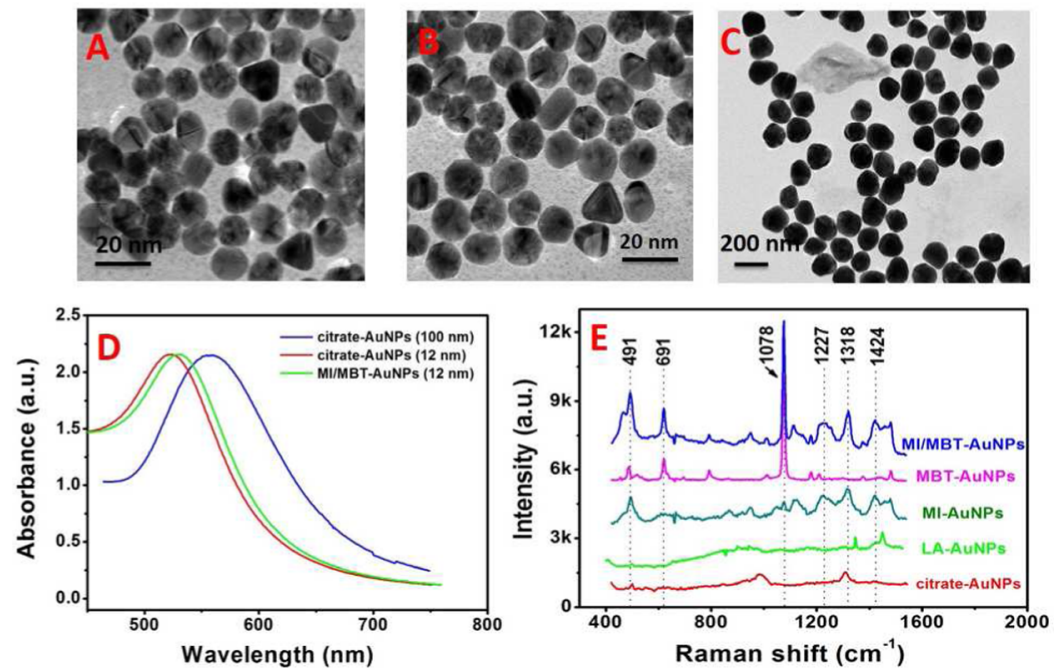

Figure 1. Characterization of AuNPs modified with different ligands. TEM images of the satellite AuNPs (A) before and (B) after modification with $\mathrm{MI} / \mathrm{MBT}$ ligands, and (C) the core AuNPs. (D) Extinction spectra comparison of different kinds of AuNPs in aqueous solution. (E) SERS spectra of AuNPs with various capping ligands.


Figure 2. Dispersion of the MI/MBT-AuNPs under various conditions. Absorbance ratio and corresponding surface charge of (A) the AuNPs after exposure to the MI/MBT solution for different times, and (B) the resultant MI/MBT-AuNPs at various $\mathrm{pH}$ values.

previously, the two nitrogen atoms in the imidazole side chain have different roles that depend on the surrounding conditions: one nitrogen is bound to hydrogen and donates its lone electron pair to the aromatic ring, while the other can act as a base and hence as a hydrogen acceptor or metal coordinator. Because of this property, the MI ligand can form $\mathrm{N}-\mathrm{H} \cdots \mathrm{N}$ intermolecular $\mathrm{HB}$ along the periphery of the AuNPs when the protonation state is regulated using an external factor, i.e., the local $\mathrm{pH}$ (Scheme 1). Figure $2 \mathrm{~B}$ reveals the $\mathrm{pH}$-responsive assembly/disassembly behavior of the MI/MBT-AuNPs. At $\mathrm{pH}$ $\geq 5.0$, the $A_{630} / A_{530}$ values of the AuNP colloid remain stable, indicating that the Coulomb repulsion from negatively charged LA on the surface of the MI/MBT-AuNPs is great enough to hinder aggregation. At $\mathrm{pH}<5.0$, owing to reduced electrostatic repulsion from protonation of the LA moiety, the $A_{630} / A_{530}$ value increases gradually, and the original well-dispersed AuNPs begin to form interconnected mesoscopic MI/MBT-AuNP objects via interparticle $\mathrm{HB}$ interactions. We also noted that once the $\mathrm{pH}$ was again adjusted to above 5.0, the MI/MBTAuNP agglomeration was redispersed swiftly, indicating that this noncovalent assembly via $\mathrm{HB}$ allows one to control the dispersion mode of the resultant MI/MBT-AuNPs in a reversible manner using an external $\mathrm{pH}$ stimulus.

3.2. Fabrication of the MI-Modified AuNP Array on a SH-Si Wafer. To create an AuNP array on SH-Si with reproducible and uniform interparticle junctions, we performed a bottom-up assembly of AuNPs on a SH-Si wafer that had a dense layer of thiol groups for stable adhesion of AuNPs to prevent loss and aggregation in real chemical reaction conditions. Subsequently, citrate-AuNPs@SH-Si was exposed to the surrounding solution to allow modification of the MI molecules. As evidenced by SEM (Figure 3A), the core AuNPs $(\sim 100 \mathrm{~nm})$ are uniformly dispersed on the substrate in close proximity to each other. An enlarged image (Figure 3B) shows
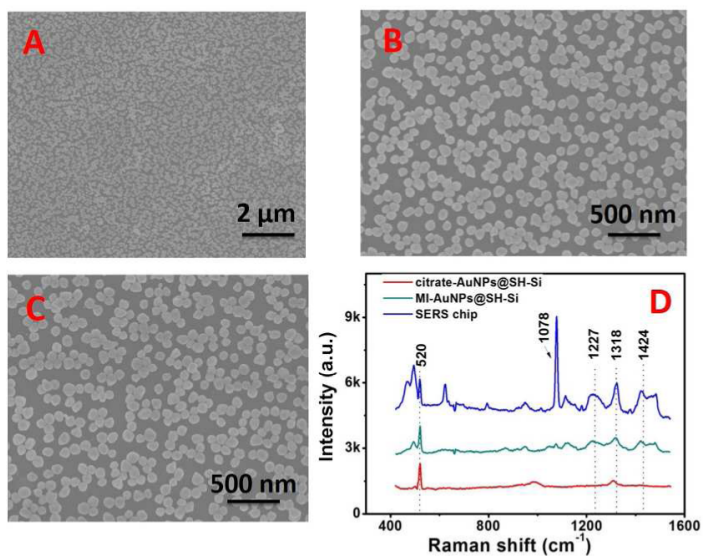

Figure 3. Characterization of AuNPs@SH-Si. (A) SEM images of citrate-AuNPs@SH-Si, (B) an enlarged image of the same sample, and (C) that of MI-AuNPs@SH-Si, as well as (D) the corresponding SERS spectra. 
that there is no evident change in the dimension and shape of these AuNPs when going from the solution phase to the surface-bound state. The AuNP array was revealed to comprise a self-assembled MI monolayer (MI-AuNPs@SH-Si) that preserved the morphology and coating density on $\mathrm{SH}-\mathrm{Si}$ after modification (Figure 3C). To verify the successful modification of MI ligands, SERS experiments were conduction with MIAuNPs@SH-Si. Notably, although the signal is weak (Figure 3D), the SERS spectrum is dominated by the vibrational modes of MI, unlike citrate-AuNPs@SH-Si.

3.3. Fabrication of the SERS Chip by Cross-Conjugation of the Satellite MI/MBT-AuNPs on MI-AuNPs@ SH-Si. To fabricate a SERS chip with enhanced sensitivity and a specific response to $\mathrm{HClO}$, we took advantage of MI-mediated $\mathrm{HB}$ formation to promote cross-conjugation between the satellite MI/MBT-AuNPs and the core MI-AuNPs on the SH$\mathrm{Si}$ substrate, while self-conjugation among the MI/MBTAuNPs themselves was prevented. Specifically, the MI monolayer significantly neutralizes the overall negative surfaces charges around AuNPs@SH-Si, thus weakening the electrostatic repulsion against the MI/MBT-AuNPs, which greatly favors the assembly of the MI/MBT-AuNPs around MIAuNPs@SH-Si via intermolecular HB interactions. As acidic condition benefit $\mathrm{HB}$ formation and the MI/MBT-AuNP colloids were mondisperse above $\mathrm{pH}$ 5.0, we chose $\mathrm{pH} 5.0$ as the assembly condition. The formed Au nanostructure provided continuous enhancement of the SERS signal (Figure 4A) over
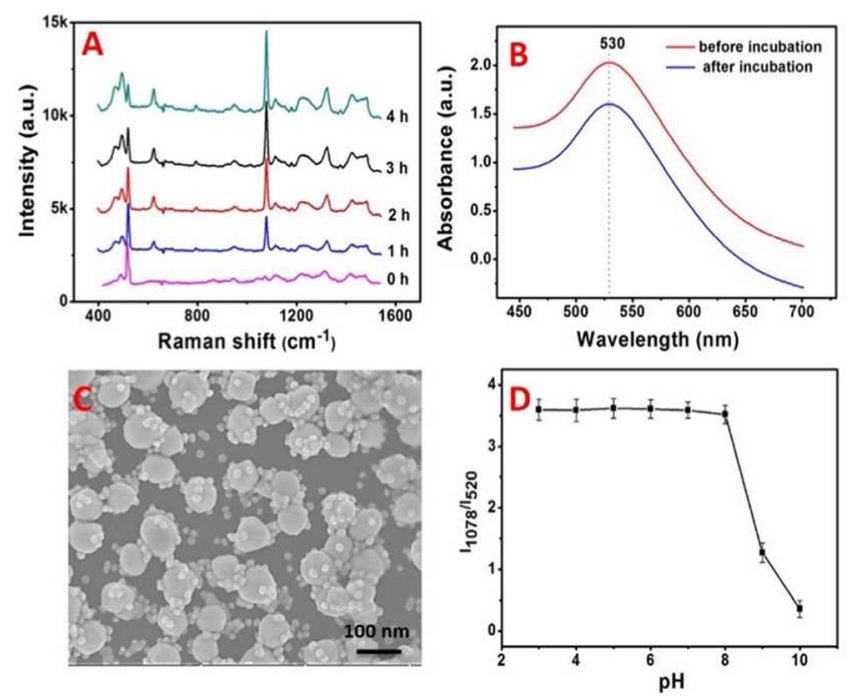

Figure 4. Optimization of the fabrication conditions for the SERS chip. (A) Signal enhancement of the SERS chip at different assembly times. (B) Comparison of the absorbance spectra of the same MI/ MBT-AuNP sample before and after incubation with MI-AuNPs@SHSi. (C) SEM image of the MI-AuNPs@SH-Si with MI/MBT-AuNPs assemble around them. (D) Relative intensity changes of the SERS chip under different $\mathrm{pH}$ conditions.

time. Finally, the maximum enhancement was obtained after incubation for $4 \mathrm{~h}$. SEM observations (Figure 4C) confirmed that the MI/MBT-AuNPs are spatially isolated from each other, but are indeed assembled on MI-AuNPs@SH-Si, which induces strong plasmonic coupling between the MI/MBT-AuNPs and the core MI-AuNPs. In contrast, little aggregation of the MI/ MBT-AuNPs occurred in the same incubation period $(4 \mathrm{~h})$, as observed in the absorbance spectra (Figure 4B), which was attributed to the sufficient repulsive force among the MI/MBTAuNPs with a zeta potential of $-11.4 \mathrm{mV}$ at $\mathrm{pH} 5.0$ (Figure 2B). Moreover, we also evaluated the signal performance of the SERS chip with different sized AuNPs ( 60, 75, 90, and $\sim 100 \mathrm{~nm})$ as core and $\sim 12 \mathrm{~nm}$ AuNPs as satellite. The comparison revealed that the SERS chip with $\sim 100 \mathrm{~nm}$ core AuNPs possess the most sensitive background signal (Figure S1 in the Supporting Information), which is optimal for "turn-off" SERS detection. To evaluate the stability of the developed SERS chip at different $\mathrm{pH}$ values, we measured the change in the relative SERS intensity at 1078 and $520 \mathrm{~cm}^{-1}\left(I_{1078} / I_{520}\right)$. The SERS chip was stable in acidic and weakly basic environments ( $\mathrm{pH} 3-8)$, indicating that it has relatively wide working $\mathrm{pH}$ range (Figure $4 \mathrm{D}$ ).

Despite relying on self-organization, the chip showed a uniform signal distribution; as shown from the SERS mapping (Figure $5 \mathrm{~A}$ ), the site-to-site relative intensity $\left(I_{1078} / I_{520}\right)$ had a
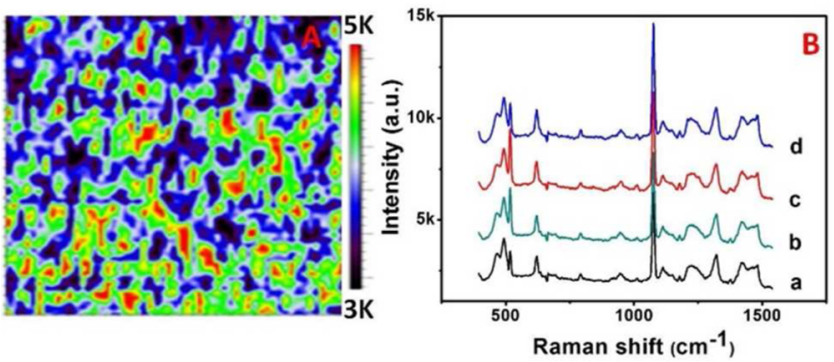

Figure 5. Assessing the uniformity of the SERS chip. (A) Twodimensional SERS mapping of the relative intensity of $I_{1078} / I_{520}$. (B) Four randomly selected SERS spectra from image A.

standard deviation of $<15 \%$ across a $200 \mu \mathrm{m} \times 200 \mu \mathrm{m}$ area of the chip. Moreover, the SERS spectra from different locations were very similar (Figure 5B), with significant structural information consistent with the MBT reporter. In addition to the enhanced SERS signal and better reproducibility, this assemble strategy also provides the chip with good stability in biological media. After incubating the chip in 10 times diluted human serum for $12 \mathrm{~h}$, the SERS signal of the chip retained more than $90 \%$ of its original intensity (Figure S2 in the Supporting Information), because of the strong $\mathrm{Au}-\mathrm{S}$ covalent linkages, as well as the stable HB interactions. The stability of the SERS signal indicates that inactivation of the chip during research and clinical applications is not a concern.

3.4. HCIO Sensing Using the SERS Chip. After achieving a stable SERS chip, we proceeded to evaluate its applicability for $\mathrm{HClO}$ sensing. We hypothesized that if the SERS chip were treated with $\mathrm{HClO}$, imidogen $(\mathrm{NH})$ in the MI ligand would be rapidly oxidized, primarily to imidazole chloramines, ${ }^{35}$ which are not capable of forming $\mathrm{NH} \cdots \mathrm{N}$ HBs, thereby leading to the disassembly of the MI/MBT-AuNPs from the MI-AuNPs@SH$\mathrm{Si}$ substrate. Notably, with increased addition of $\mathrm{HClO}$, the shape of the SERS spectra of the chips did not change significantly, but a dose-dependent decrease in intensity was observed (Figure 6A), with a minimum value a $1.5 \mu \mathrm{mol} \mathrm{L}^{-1}$ $\mathrm{HClO}$. This reaction process was also monitored by SEM, and the results are shown in Figures $6 \mathrm{~B}-\mathrm{D}$. The SERS chip without $\mathrm{HClO}$ treatment had the highest assemble efficiency, but with the increasing $\mathrm{HClO}$ concentration, the average number of MI/ MBT-AuNPs around MI-AuNPs@SH-Si decreased gradually, indicating that the damping of SERS signal can be attributed to the loss of hot-spots on the SERS chip. Nevertheless, these 


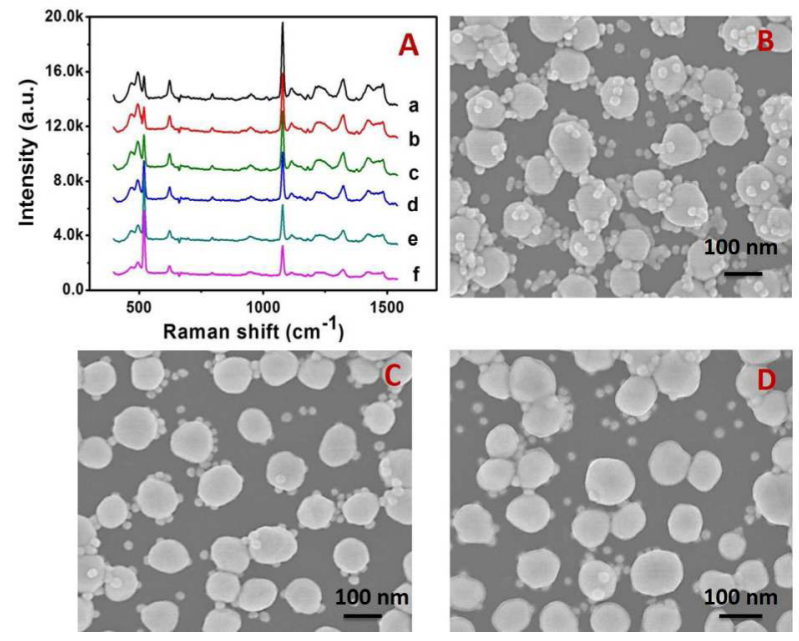

Figure 6. HClO sensing process of the SERS chip. (A) Signal change of the SERS chip treated with various concentrations of $\mathrm{HClO}(\mathrm{a}-\mathrm{f}) \mathrm{f}$, $10,20,30,40$, and $\left.50 \mu \mathrm{mol} \mathrm{L}{ }^{-1}\right)$. SEM images of the SERS chip after reacting with different concentrations of $\mathrm{HClO}$ : (B) 0 , (C) 30, and (D) $50 \mu \mathrm{mol} \mathrm{L}-1$.

preliminary results suggest the potential of the developed chip for use in a SERS assay to detect HClO.

The detection conditions were optimized to enhance the method sensitivity. As $\mathrm{HClO}$ has a greater oxidization capability and decomposes more rapidly in strongly acidic solutions, neutral and acidic conditions should be more suitable for $\mathrm{HClO}$ detection. On the other hand, the SERS chip is stable in the $\mathrm{pH}$ range of 3.0 to 8.0 , as discussed above (Figure 4D). As a compromise, we chose $\mathrm{pH} 6.0$ for this assay. To optimize the reaction time, the SERS spectra after treatment with $\mathrm{HClO}$ was collected at different time intervals. The signal decreased within the initial $30 \mathrm{~min}$, and then leveled off (Figure S3 in the SI). Therefore, a reaction time of $30 \mathrm{~min}$ was chosen to ensure complete reaction. Under the optimum conditions, a linear correlation equation of $I_{1078} / I_{520}=4.21-0.07 \mathrm{C}_{\mathrm{HClO}}$ was obtained in the range of $1.5-50.0 \mu \mathrm{mol} \mathrm{L}^{-1} \mathrm{HClO}(\mathrm{R}=$ 0.989) (Figure 7A). The LOD was calculated to be $1.2 \mu \mathrm{mol}$ $\mathrm{L}^{-1}$ based on the criterion $3 \mathrm{~s} / \mathrm{m}$, where $\mathrm{m}$ is the slope in the linear range and $s$ is the standard deviation of 10 spectra of the blank. To verify the specificity of this assay, we evaluated the $I_{1078} / I_{520}$ values in the presence of $1.0 \mathrm{mmol} \mathrm{L}^{-1}$ of inorganic interfering substances. It is revealed that common ions such as $\mathrm{Na}^{+}, \mathrm{K}^{+}, \mathrm{Ca}^{2+}, \mathrm{Mg}^{2+}, \mathrm{Al}^{3+}, \mathrm{Cl}^{-}, \mathrm{Br}^{-}, \mathrm{SO}_{4}{ }^{2-}, \mathrm{CO}_{3}{ }^{2-}, \mathrm{NO}_{3}{ }^{2-}$,
$\mathrm{PO}_{4}{ }^{3-}$ (data not shown) and some strong oxidants $\left(\mathrm{ClO}_{3}{ }^{-}\right.$, $\mathrm{ClO}_{4}^{-}$, and $\mathrm{NO}_{2}{ }^{-}$in Figure $7 \mathrm{~B}$ ), induce no significant effects on the assay. However, some transition metal ions such as $\mathrm{Cu}^{2+}$ and $\mathrm{Ni}^{2+}$ would induce significant interference (as shown in Figure S4 in the Supporting Information), because of their complexing with imidazole moieties and therefore breaking the HB linkage within core and satellite AuNPs. These results suggest that although the physiological concentration of $\mathrm{Cu}^{2+}$ and $\mathrm{Ni}^{2+}$ would not affect the detection of $\mathrm{HClO}$ in human serum analysis, extra treatments such as utilizing masking agents to eliminate metal interferences should be adopted when the SERS chip is used to detect $\mathrm{HClO}$ in samples rich in $\mathrm{Cu}^{2+}$ and $\mathrm{Ni}^{2+}$. Furthermore, an additional control experiment by competitive ROS/RNS species was conducted and no apparent interferences were observed (Figure 7B). These results demonstrate that the SERS chip is a selective sensor for $\mathrm{HClO}$ assay.

To validate its applicability in real sample analysis, the SERS chip was used to determine $\mathrm{HClO}$ in tap water and diluted human serum. Although $\mathrm{HClO}$ was present in tap water at levels around $2 \mu \mathrm{mol} \mathrm{L}{ }^{-1}$, the $\mathrm{HClO}$ content detected in the human serum sample was below the detection limit. To further verify the proposed method, we tested the spiked recovery of $\mathrm{HClO}$. The recoveries for all of these samples (Table 1) were in the range of $96-104 \%$, indicating the applicability of this assay for samples with complex matrices.

\section{CONCLUSIONS}

In summary, we have designed and constructed a stable SERS chip with high selectivity and sensitivity for quantifying minute amounts of $\mathrm{HClO}$ in tap water and human serum. This SERS chip offers uniform and reproducible signals owing to the plasmon coupling between AuNPs with a dense collection of hot spots. Our work marks an improvement in terms of ease and economic fabrication of SERS chips, and avoids the complicated operation and tedious organic synthesis required for other methods, making it a promising and attractive candidate to serve as an easy and applicable way to test for $\mathrm{HClO}$ in drinking water or various biological samples.

\section{ASSOCIATED CONTENT}

\section{S Supporting Information}

The preparation of $\mathrm{SH}-\mathrm{Si}$ and various ROS stock solutions, detailed SERS experimental conditions. The Supporting Information is available free of charge on the ACS Publications website at DOI: 10.1021/acsami.5b04449.
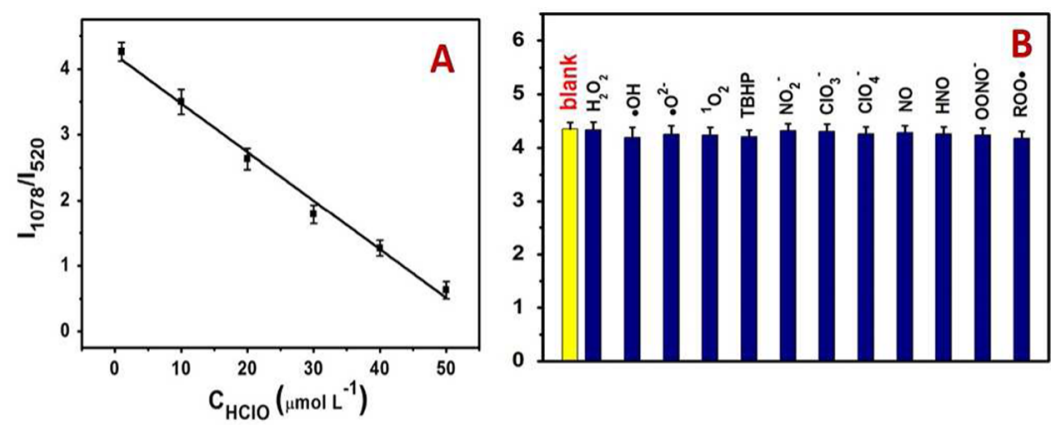

Figure 7. Analytical performance of the developed SERS chip. (A) Linear plot of $I_{1078} / I_{520}$ as a function of HClO concentration. The error bars represent the standard deviations for five replicated experiments. (B) Signal response of the SERS chip in the presence of various ROS/RNS and oxidants at $1.0 \mathrm{mmol} \mathrm{L}^{-1}$. 


\section{AUTHOR INFORMATION}

\section{Corresponding Author}

* E-mail: jfliu@rcees.ac.cn.

Notes

The authors declare no competing financial interest.

\section{ACKNOWLEDGMENTS}

This work was supported by the Strategic Priority Research Program of the Chinese Academy of Sciences (XDB14020101), and the National Natural Science Foundation of China (21207142, 21321004).

\section{REFERENCES}

(1) Morris, J. C. The Acid Ionization Constant of $\mathrm{HOCl}$ from 5 to 35. J. Phys. Chem. 1966, 70, 3798-3805.

(2) Harrison, J. E.; Schultz, J. Studies on the Chlorinating Activity of Myeloperoxidase. J. Biol. Chem. 1976, 251, 1371-1374.

(3) Dickinson, B. C.; Srikun, D.; Chang, C. J. MitochondrialTargeted Fluorescent Probes for Reactive Oxygen Species. Curr. Opin. Chem. Biol. 2010, 14, 50-56.

(4) Jiang, Q.; Xiao, N.; Shi, P.; Zhu, Y.; Guo, Z. Design of Artificial Metallonucleases with Oxidative Mechanism. Coord. Chem. Rev. 2007, 251, 1951-1972.

(5) Hawkins, C. L.; Davies, M. J. Hypochlorite-Induced Damage to Proteins: Formation of Nitrogen-Centered Radicals from Lysine Residues and Their Role in Protein Fragmentation. Biochem. J. 1998, 332, 617-625.

(6) Brennan, M. L.; Anderson, M. M.; Shih, D. M.; Qu, X. D.; Wang, X.; Mehta, A. C.; Lim, L. L.; Shi, W.; Hazen, S. L.; Jacob, J. S. Increased Atherosclerosis in Myeloperoxidase-Deficient Mice. J. Clin. Invest. 2001, 107, 419-430.

(7) Malle, E.; Buch, T.; Grone, H. J. Myeloperoxidase in Kidney Disease. Kidney Int. 2003, 64, 1956-1967.

(8) Hammerschmidt, S.; Vogel, T.; Jockel, S.; Gessner, C.; Seyfarth, H. J.; Gillissen, A.; Wirtz, H. Protein Kinase C Inhibition Attenuates Hypochlorite-Induced Acute Lung Injury. Respir. Med. 2007, 101, $1205-1211$.

(9) Soldatkin, A. P.; Gorchkov, D. V.; Martelet, C.; Jaffrezic-Renault, N. New Enzyme Potentiometric Ssensor for Hypochlorite Species Detection. Sens. Actuators, B 1997, 43, 99-104.

(10) Gallina, A.; Pastore, P.; Magno, F. The Use of Nitrite Ion in the Chromatographic Determination of Hypochlorite Ion and Of Traces of Chlorite and Chlorate Ions. Analyst 1999, 124, 1439-1442.

(11) Watanabe, T.; Idehara, T.; Yoshimura, Y.; Nakazawa, H. Simultaneous Determination of Chlorine Dioxide and Hypochlorite in Water by High-Performance Liquid Chromatography. J. Chromatogr. A 1998, 796, 397-400.

(12) Lou, X. D.; Zhang, Y.; Li, Q. Q.; Qin, J. G.; Li, Z. A Highly Specific Rhodamine-Based Colorimetric Probe for Hypochlorites: A New Sensing Strategy and Real Application in Tap Water. Chem. Commun. 2011, 47, 3189-3191.

(13) Hou, J. T.; Wu, M. Y.; Li, K.; Yang, J.; Yu, K. K.; Xie, Y. M.; Yu, X. Q. Mitochondria-Targeted Colorimetric and Fluorescent Probes for Hypochlorite and Their Applications for in vivo Imaging. Chem. Commun. 2014, 50, 8640-8643.

(14) Kenmoku, S.; Urano, Y.; Kojima, H.; Nagano, T. Development of a Highly Specific Rhodamine-Based Fluorescence Probe for Hypochlorous Acid and Its Application to Real-Time Imaging of Phagocytosis. J. Am. Chem. Soc. 2007, 129, 7313-7318.

(15) Koide, Y.; Urano, Y.; Hanaoka, K.; Terai, T.; Nagano, T. Development of a Si-Rhodamine-Based Far-Red to Near-Infrared Fluorescence Probe Selective for Hypochlorous Acid and Its Applications for Biological Imaging. J. Am. Chem. Soc. 2011, 133, $5680-5682$.

(16) Zhou, Y.; Pei, W.; Wang, C.; Zhu, J.; Wu, J.; Yan, Q.; Huang, L.; Huang, W.; Yao, C.; Say, J.; Loo, C.; Zhang, Q. Rhodamine-Modified Upconversion Nanophosphors for Ratiometric Detection of Hypo- chlorous Acid in Aqueous Solution and Living Cells. Small 2014, 10, $3560-3567$.

(17) Wang, Y.; Yan, B.; Chen, L. SERS Tags: Novel Optical Nanoprobes for Bioanalysis. Chem. Rev. 2013, 113, 1391-1428.

(18) Cao, Y. C.; Jin, R.; Mirkin, C. A. Nanoparticles with Raman Spectroscopic Fingerprints for DNA and RNA Detection. Science 2002, 297, 1536-1540.

(19) Li, M.; Cushing, S. K.; Zhang, J.; Suri, S.; Evans, R.; Petros, W. P.; Gibson, L. F.; Ma, D.; Liu, Y.; Wu, N. Three-Dimensional Hierarchical Plasmonic Nano-Architecture Enhanced Surface-Enhanced Raman Scattering Immunosensor for Cancer Biomarker Detection in Blood Plasma. ACS Nano 2013, 7, 4967-4976.

(20) Liu, T. Y.; Tsai, K. T.; Wang, H. H.; Chen, Y.; Chen, Y. H.; Chao, Y. C.; Chang, H. H.; Lin, C. H.; Wang, J. K.; Wang, Y. L. Functionalized Arrays of Raman-Enhancing Nanoparticles for Capture and Culture-Free Analysis of Bacteria in Human Blood. Nat. Commun. 2011, 2, 538-541.

(21) Kang, T.; Yoo, S. M.; Yoon, I.; Lee, S. Y.; Kim, B. Patterned Multiplex Pathogen DNA Detection by Au Particle-on-Wire SERS Sensor. Nano Lett. 2010, 10, 1189-1193.

(22) Zhu, Y.; Kuang, H.; Xu, L.; Ma, W.; Peng, C.; Hua, Y.; Wang, L.; $\mathrm{Xu}, \mathrm{C}$. Gold Nanorod Assembly Based Approach to Toxin Detection by SERS. J. Mater. Chem. 2012, 22, 2387-2391.

(23) Kasera, S.; Biedermann, F.; Baumberg, J. J.; Scherman, O. A.; Mahajan, S. Quantitative SERS Using the Sequestration of Small Molecules inside Precise Plasmonic Nanoconstructs. Nano Lett. 2012, $12,5924-5928$

(24) Salinas, Y.; Martinez-Manez, R.; Marcos, M. D.; Sancenon, F.; Costero, A. M.; Parra, M.; Gil, S. Optical Chemosensors and Reagents to Detect Explosives. Chem. Soc. Rev. 2012, 41, 1261-1296.

(25) Alvarez-Puebla, R. A.; Liz-Marzan, L. M. SERS Detection of Small Inorganic Molecules and Ions. Angew. Chem., Int. Ed. 2012, 51, 11214-11223.

(26) Bishnoi, S. W.; Rozell, C. J.; Levin, C. S.; Gheith, M. K.; Johnson, B. R.; Johnson, D. H.; Halas, N. J. All-Optical Nanoscale pH Meter. Nano Lett. 2006, 6, 1687-1692.

(27) Wang, W.; Yin, Y.; Tan, Z.; Liu, J. Coffee-Ring Effect-Based Simultaneous SERS Substrate Fabrication and Analyte Enrichment for Trace Analysis. Nanoscale 2014, 6, 9588-9593.

(28) Lee, S. J.; Moskovits, M. Visualizing Chromatographic Separation of Metal Ions on a Surface-Enhanced Raman Active Medium. Nano Lett. 2011, 11, 145-150.

(29) Zhao, Y.; Newton, J. N.; Liu, J.; Wei, A. DithiocarbamateCoated SERS Substrates: Sensitivity Gain by Partial Surface Passivation. Langmuir 2009, 25, 13833-13839.

(30) Krpetic, Z.; Guerrini, L.; Larmour, I. A.; Reglinski, R.; Faulds, K.; Graham, D. Importance of Nanoparticle Size in Colorimetric and SERS-Based Multimodal Trace Detection of $\mathrm{Ni}$ (II) Ions with Functional Gold Nanoparticles. Small 2012, 8, 707-714.

(31) Tsoutsi, D.; Montenegro, J. M.; Dommershausen, F.; Koert, U.; Liz-Marzan, L. M.; Parak, W. J.; Alvarez-Puebla, R. A. Quantitative Surface-Enhanced Raman Scattering Ultradetection of Atomic Inorganic Ions: The Case of Chloride. ACS Nano 2011, 5, 7539-7546.

(32) Pienpinijtham, P.; Han, X. X.; Ekgasit, S.; Ozaki, Y. Highly Sensitive and Selective Determination of Iodide and Thiocyanate Concentrations Using Surface-Enhanced Raman Scattering of StarchReduced Gold Nanoparticles. Anal. Chem. 2011, 83, 3655-3662.

(33) Senapati, D.; Dasary, S. S. R.; Singh, A. K.; Senapati, T.; Yu, H.; Ray, P. C. A Label-Free Gold-Nanoparticle-Based SERS Assay for Direct Cyanide Detection at the Parts-per-Trillion Level. Chem. - Eur. J. 2011, 17, 8445-8451.

(34) Alvarez-Puebla, R. A.; Liz-Marzán, L. M. SERS Detection of Small Inorganic Molecules and Ions. Angew. Chem., Int. Ed. 2012, 51, 11214-11223.

(35) Pattison, D. I.; Davies, M. J. Evidence for Rapid Inter- and Intramolecular Chlorine Transfer Reactions of Histamine and Carnosine Chloramines: Implications for the Prevention of Hypochlorous-Acid-Mediated Damage. Biochemistry 2006, 45, 8152-8162. 
(36) Grabar, K. C.; Freeman, R. G.; Hommer, M. B.; Natan, M. J. Preparation and Characterization of Au Colloid Monolayers. Anal. Chem. 1995, 67, 735-743.

(37) Lin, S. Y.; Tsai, Y. T.; Chen, C. C.; Lin, C. M.; Chen, C. H. Two-Step Functionalization of Neutral and Positively Charged Thiols onto Citrate-Stabilized Au Nanoparticles. J. Phys. Chem. B 2004, 108, 2134-2139.

(38) Storhoff, J. J.; Elghanian, R.; Mucic, R. C.; Mirkin, C. A.; Letsinger, R. L. One-Pot Colorimetric Differentiation of Polynucleotides with Single Base Imperfections Using Gold Nanoparticle Probes. J. Am. Chem. Soc. 1998, 120, 1959-1964.

(39) Bastus, N. G.; Comenge, J.; Puntes, V. Kinetically Controlled Seeded Growth Synthesis of Citrate-Stabilized Gold Nanoparticles of up to $200 \mathrm{~nm}$ : Size Focusing versus Ostwald Ripening. Langmuir 2011, $27,11098-11105$. 